OPEN ACCESS

Edited by:

Suraj Bahadur Thapa,

University of Oslo, Norway

Reviewed by: Krishnamachari Srinivasan,

St. John's Research Institute, India Leire Aperribai,

University of the Basque

Country, Spain

*Correspondence:

Nagahide Takahashi

n-taka@med.nagoya-u.ac.jp

${ }^{\dagger}$ These authors share first authorship

Specialty section: This article was submitted to Mood Disorders,

a section of the journal

Frontiers in Psychiatry

Received: 16 November 2021 Accepted: 31 December 2021 Published: 24 January 2022

Citation:

Nakamura Y, Takahashi N, Yamauchi A, Morikawa M, Okada T and Ozaki N (2022) Perceived Social

Support Partially Mediates the Impact of Temperament and Character on Postpartum Depression

Front. Psychiatry 12:816342

doi: 10.3389/fpsyt.2021.816342

\section{Perceived Social Support Partially Mediates the Impact of Temperament and Character on Postpartum Depression}

\author{
Yukako Nakamura ${ }^{1 \dagger}$, Nagahide Takahashi $^{2 \star \dagger}$, Aya Yamauchi ${ }^{1,3}$, Mako Morikawa ${ }^{1}$, \\ Takashi Okada ${ }^{1,4}$ and Norio Ozaki ${ }^{1,2}$ \\ ${ }^{1}$ Department of Psychiatry, Nagoya University Graduate School of Medicine, Nagoya, Japan, ${ }^{2}$ Department of Child and \\ Adolescent Psychiatry, Nagoya University Graduate School of Medicine, Nagoya, Japan, ${ }^{3}$ Psychiatry/Child and Adolescent \\ Psychiatry, Nagoya University Hospital, Nagoya, Japan, ${ }^{4}$ Department of Developmental Disorders, National Center of \\ Neurology and Psychiatry, Kodaira, Japan
}

Introduction: Temperament and character of pregnant women, especially harm avoidance (HA) and self-directedness (SD) have been identified as risk factors for postpartum depression, in addition to poor social support. However, the relationship between these personality traits and social support for depressive symptoms after delivery has not been examined.

Methods: Data were extracted from a prospective cohort survey on pregnant women conducted in Nagoya, Japan that included the Temperament and Character Inventory (TCl), the Social Support Questionnaire (J-SSQ), and the Edinburgh Postnatal Depression Scale (EPDS) at approximately week 25 and 1 month postpartum. A mediation analysis using structural equation modeling (SEM) was used to test if social support in pregnancy is a mediator between personality traits and postpartum depressive symptoms.

Results: Thousand five hundred and fifty-nine women were included in the analysis. Both harm avoidance and SD were significantly associated with depressive symptoms (total effect: $\beta$ [SE], 0.298 [0.041], $P<0.001$ for harm avoidance; total effect: $\beta$ [SE], -0.265 [0.067], $P<0.001$ for SD). Mediation analysis showed that the effect of harm avoidance on depressive symptoms was partially mediated by low social support (direct effect: $\beta$ [SE], 0.193 [0.004], $P<0.001$; indirect effect: $\beta$ [SE], 0.082 [0.034], $P=0.015$ ). Self-directedness on depressive symptoms was not found to be mediated by low social support.

Conclusion: Results indicate that poor social support worsens depressive symptoms in women with high HA during pregnancy. Limitations include a possible selection bias due to the limited target facilities; most variables being evaluated based on self-report questionnaires, and different number of samples available for analysis between harm avoidance and SD.

Keywords: cohort study, depression, postpartum, personality, perceived social support, mediation analysis 


\section{INTRODUCTION}

Postpartum depression, which has an approximate prevalence of $10-15 \%$ in mothers (1-3), can lead to maternal suicide (4-6) and the developmental failure of the child (7-9). Thus, identification of the risk for postpartum depression, and protective factors against its development, is critical $(10,11)$. It has been pointed out that the involvement of temperament and personality in the development of depression using various questionnaires or inventories (12-14). Among them, research between depression and temperament and character $(13,15)$ using the Temperament and Character Inventory (TCI) developed by Cloninger et al. (16) has been extensively studied (13-18) and a longitudinal study has demonstrated that TCI is clinically useful for predicting future risk of developing depression (19).

Temperament and Character Inventory is based on a sevendimensional model consisting of temperament (four dimensions) and personality (three dimensions). The four dimensions of temperament are Novelty Seeking (NS), Harm Avoidance (HA), Reward Dependence (RD), and persistence (P), and the three dimensions of personality are, self-directedness (SD), Cooperativeness (C), and Self-Transcendence (ST) (20). Of the seven dimensions proposed by Cloninger et al., HA and SD have been repeatedly reported to be particularly associated with depression $(13,17,18,21,22)$. Cloninger et al. proposed Temperament being stable, with a biological basis, and Character being acquired $(16,20)$. However, subsequent research confirmed the state-dependence of temperament $(23,24)$, and recently the distinction between the constructs of temperament and personality has been called into question (25).

Previously we found that scores of HA increase with the severity of depressive symptoms (24), and that HA predicts the development of postpartum depression (26). Earlier studies of perinatal women pointed to an association between HA and SD with depression during pregnancy $(27,28)$, as well as postpartum depression, using relatively small samples (29). It has also been reported that personality, measured 2-3 days after delivery, predicts depression at 8 and 32 weeks after delivery (30). Taken together, it is possible that assessment of personality of perinatal women is important as it may identify pregnant women at risk for development of postpartum depression. However, there have been no studies that have evaluated the association of personality traits in pregnancy with the risk of postpartum depression using a large sample size.

In addition to the above, social support during pregnancy has been shown to be protective against postpartum depression (31). However, it has not been elucidated whether social support is protective in women with high-risk personality traits for postpartum depression. Using structural equation modeling (SEM), Kendler et al. examined factors involved in depression for women, and also examined social support (32), but they evaluated social support in late adolescence, not in the perinatal period. In order to examine risk factors and protective factors for perinatal depression, it is necessary to evaluate personality traits as well as social support in the perinatal period.

In the present study, therefore, we used SEM to clarify the relationship between personality traits, social support, and postpartum depression using data from our prospective perinatal cohort and identify protective factors in subjects with high-risk personality traits for the development of postpartum depression.

\section{MATERIALS AND METHODS}

\section{Design}

Data from this study were extracted from a prospective cohort study carried out in Nagoya, Japan, from August 2004 to March 2020. Three questionnaires, the Edinburgh Postnatal Depression Scale (EPDS), TCI, and Japanese version of Social Support Questionnaire (J-SSQ) were used to measure depressive symptoms and related factors. The socio-demographic variables included age, partner's age, and number of children. Participants answered the questionnaire during early pregnancy (T1: around 25 weeks of gestation) which included the EPDS, TCI, and JSSQ. One month after delivery (Q2), the EPDS, were filled out and the questionnaire was mailed back. This study followed the STrengthening the Reporting of OBservational studies in Epidemiology (STROBE) reporting guideline.

\section{Participants}

Participants were recruited from a general hospital, two obstetric and gynecological hospitals and a university hospital. Pregnant women participating in prenatal classes before the 25th week of pregnancy were invited to participate if they were aged 20 years or older and able to read and write Japanese. Since the age of adulthood in Japan is 20 years, we excluded pregnant women under the aged 20 years in this study.

\section{Ethical Considerations}

A written informed consent was obtained from all those who agreed to participate in the study. The study protocol was approved by the Ethics Committee of the Nagoya University Graduate School of Medicine and all study procedures met the Committee's guidelines and regulations. All study protocols were in accordance with the 1964 Helsinki Declaration and its later amendments.

\section{Measurements TCI}

Personality traits including HA and SD were measured using the TCI. The TCI is a self-report questionnaire consisted of 125 items that look at four dimensions of temperament (novelty seeking, $\mathrm{HA}$, reward dependence, and persistence) and three dimensions of character (SD, cooperativeness, and self-transcendence). We used the Japanese version of the TCI-125, which includes 125 questions covering 20 items pertaining to $\mathrm{HA}$ (33). The reliability and validity of the Japanese version of TCI-125 have been previously confirmed $(34,35)$. Harm avoidance scores ranged from 0 to 20 and SD scores ranged from 0 to 25. Harm avoidance and SD in the early pregnancy (around week 25) were used for the analysis. We started assessing the HA in August 2004, and SD in April 2011. 


\section{J-SSQ}

We used the J-SSQ (36) to measure perceived social support among pregnant women in the present study. The J-SSQ has two factors: "Number of Persons" and "Satisfaction Rating" (31). "Number of Person" reflects the average number of persons the participants perceive to be available to provide social support to them. "Satisfaction Rating" reflects an individual's average degree of satisfaction for the perceived support to be available (31). The reliability and validity of J-SSQ has been confirmed in the pregnancy and postpartum periods (36). The J-SSQ in the early pregnancy (around week 25) was used for the analysis.

\section{EPDS}

The EPDS is a self-administered questionnaire developed by Cox et al. for the screening of postpartum depression. EPDS comprises 10 items and was scored on a four-point scales with total scores ranging from 0 to 30 (37). The Japanese version of the EPDS by Okano et al. (38) was used in this study and scores above nine were utilized for the screening of major depressive episodes (sensitivity, 82\%; specificity, 95\%) (39, 40). Participants' depressive symptoms were evaluated using the EPDS 1 month after birth.

\section{Statistical Analyses}

The statistical analyses were performed in Stata, version 16. To test if social support is a mediator between personality traits and postpartum depressive symptoms, we performed SEM analysis with maximum likelihood. Missing values were estimated by full information maximum likelihood method (FIML). The analysis of the outcome (total EPDS scores) included all observed and latent variables in a single step. The medsem command in Stata was used to estimate indirect effects in the mediation model (41). We used the Monte Carlo method to test the significance of the indirect effects as described (42). Comparative fit index (CFI) and root mean squared error of the approximation (RMSEA) were used to evaluate a goodness of fit for each model.

\section{RESULTS}

\section{Demographics}

In total, 1,559 pregnant women were included in the analysis who submitted valid questionnaires at around the 25th week of pregnancy. The average age of the participants was $32.4 \pm 4.6$ years $(n=1,542)$. There were $1,138(72.9 \%)$ primiparas, 381 (24.4\%) multiparas, and 40 unanswered about parity.

\section{Correlation Between Variables}

Table 1 shows the summary of all the variables and Table 2 shows the correlations between the variables. The EPDS score at 1 month after delivery was positively correlated with HA $(r=0.259, p<0.01)$ and negatively correlated with SD $(r=-0.344, p<0.01)$, J-SSQ Number $(r=-0.111, p<0.01)$, and Satisfaction $(r=-0.097, p<0.01)$. The recruitment process was described in Figure 1.

\section{Mediation Analysis}

Figure 2 illustrates the model that shows the association between $\mathrm{HA}$ and EPDS scores (CFI $=1.00$, RMSEA $<0.001)$. Harm
TABLE 1 | Summary of measured variables.

\begin{tabular}{lcccc}
\hline & $n$ & Means & $\begin{array}{c}\text { Standard } \\
\text { deviation }\end{array}$ & Skewness \\
\hline Age & & & 4.6 & 0.04 \\
T1 (TCl) harm avoidance & 1,542 & 32.4 & 4.5 & -0.32 \\
T1 (TCl) self-directedness & 1,523 & 11.6 & 4.7 & -0.95 \\
T1 (J-SSQ) number of supporters & 1,539 & 3.9 & 2.2 & 3.60 \\
T1 (J-SSQ) satisfaction with support & 1,513 & 4.9 & 1.2 & -1.92 \\
T2 EPDS total score & 1,416 & 5.1 & 4.7 & 1.27 \\
\hline
\end{tabular}

T1, Time point 1 (around 25 weeks of pregnancy); T2, Time point 2 (1 month after delivery); $\mathrm{TCl}$, Temperament and Character Inventory; J-SSQ, Japanese version of the Social Support Questionnaire; EPDS, Edinburgh Postnatal Depression Scale.

TABLE 2 | Correlations between variables.

\begin{tabular}{lccccc}
\hline & T1 HA & T1 SD & T1 NS & T1 SS & T2 EPDS \\
\hline T1 HA_TCl & 1.000 & & & & \\
T1 SD_TCl & $-0.543^{\star *}$ & 1.000 & & & \\
T1 NS_J-SSQ & $-0.226^{\star \star}$ & $0.262^{\star \star}$ & 1.000 & & \\
T1 SS_J-SSQ & $-0.139^{\star *}$ & $0.203^{\star \star}$ & $0.154^{\star \star}$ & 1.000 & \\
T2 EPDS & $0.259^{* *}$ & $-0.344^{\star *}$ & $-0.111^{\star \star}$ & $-0.097^{\star *}$ & 1.000
\end{tabular}

T1, Time point 1(around 25 weeks of gestation); T2, time point 2 (1 month after delivery); HA, Harm Avoidance; SD Self-Directedness; TCI, Temperament and Character Inventory; NS, Number of supporters; SS, Satisfaction for support; J-SSQ, Japanese version of the Social Support Questionnaire; EPDS, Edinburgh Postnatal Depression Scale. ${ }^{* *} p<0.01$

avoidance is significantly associated with EPDS scores (total effect: $\beta$ [SE], 0.298 [0.041], $P<0.001)$. Mediation analysis showed that the effect of HA on depressive symptoms was partially mediated by low social support (direct effect: $\beta$ [SE], 0.193 [0.004], $P<0.001$; indirect effect: $\beta$ [SE], 0.082 [0.034], $P=0.015)$, indicating that $29.8 \%$ of the total effect of HA on EPDS scores was mediated through low social support.

Figure 3 illustrates the model that shows the association between SD and EPDS scores (CFI $=1.00$, RMSEA $<0.001$ ). Self-directedness is significantly associated with EPDS scores (total effect: $\beta$ [SE], -0.544 [0.067], $P<0.001$ ). Mediation analysis showed that the effect of SD on depressive symptoms was not mediated by low social support (direct effect: $\beta$ [SE], -0.265 [0.248], $P<0.001$; indirect effect: $\beta$ [SE], 0.279 [0.000], $P=0.094)$.

\section{DISCUSSION}

In the present study, we confirmed that personality traits during pregnancy predict the depressive symptomatology at 1 month postpartum. Additionally, we identified social support during pregnancy as a mediator for the severity of depressive symptoms in women at high risk for postpartum depression, which could be a potential target for intervention to protect the development of the illness. 


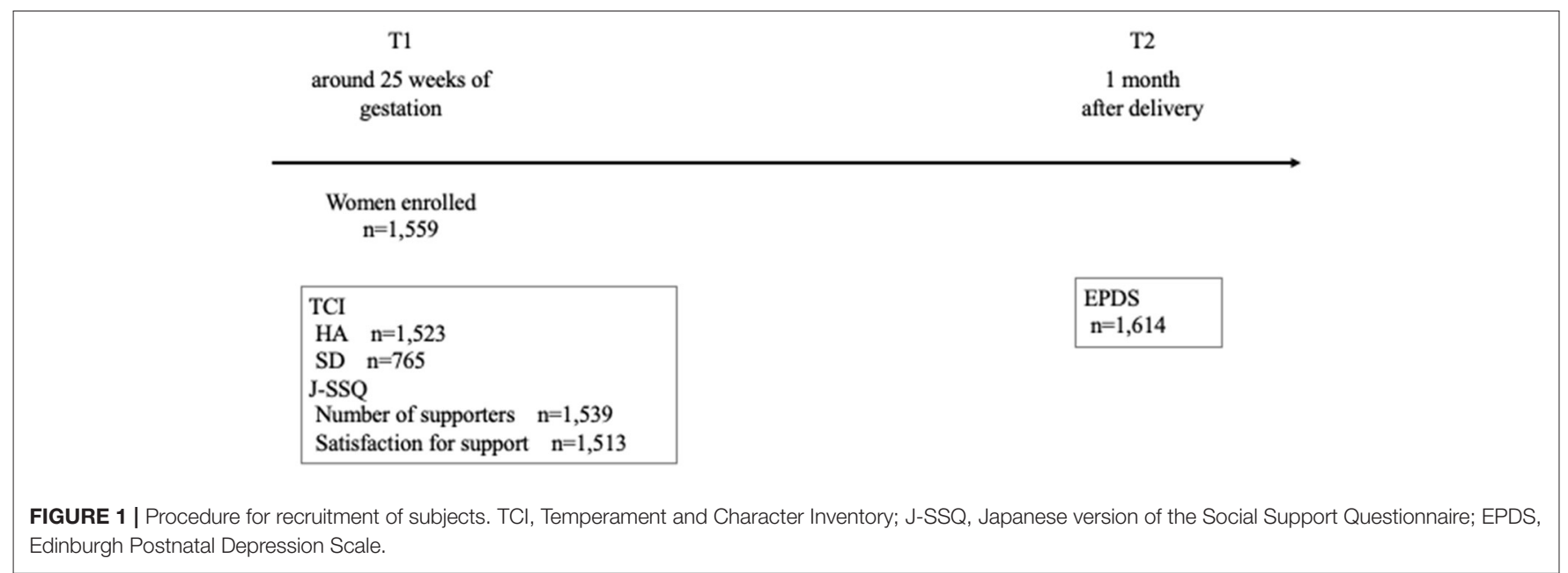

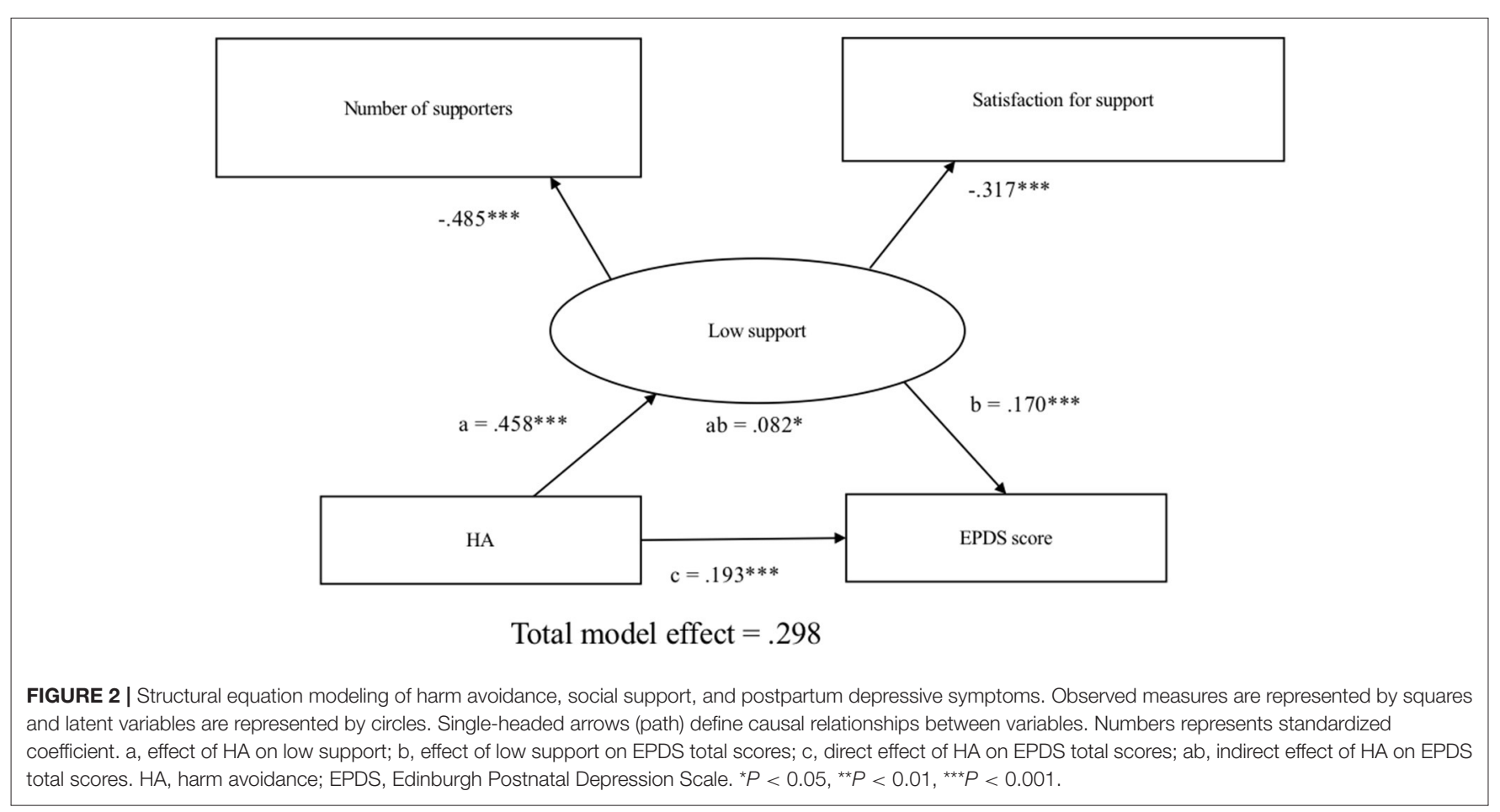

Our results showed that high $\mathrm{HA}$ and low SD around 25 weeks' gestation are associated with higher EPDS scores in the first month of postpartum, which successfully replicates previous studies of postpartum depression using relatively small sample sizes (27-29). In one of the previous results of our cohort study [Kubota et al. (26)], we reported that high HA during pregnancy predicted postpartum depression. However, HA during pregnancy did not predict postpartum depression in Furumura et al. (24). One of the possible reasons for this discrepancy is that the number of samples was small in Furumura's report, where only 99 participants responded for HA items. In this study, we expanded the sample size and confirmed that both $\mathrm{HA}$ and SD during pregnancy predict postpartum depression.

We have also confirmed that social support during pregnancy is a protective factor for postpartum depression. This result was consistent with our previous papers $(31,43-45)$. The mediation analysis showed that social support could be a partial mediator for postpartum depressive symptoms in high HA subjects, but not in low SD subjects. One possible explanation is the use of different coping strategies between high HA and low SD subjects, which is supported by a recent study using office workers (46). This study suggests low HA is associated with problem-focused coping skills, whereas low SD is associated with emotion-focused 


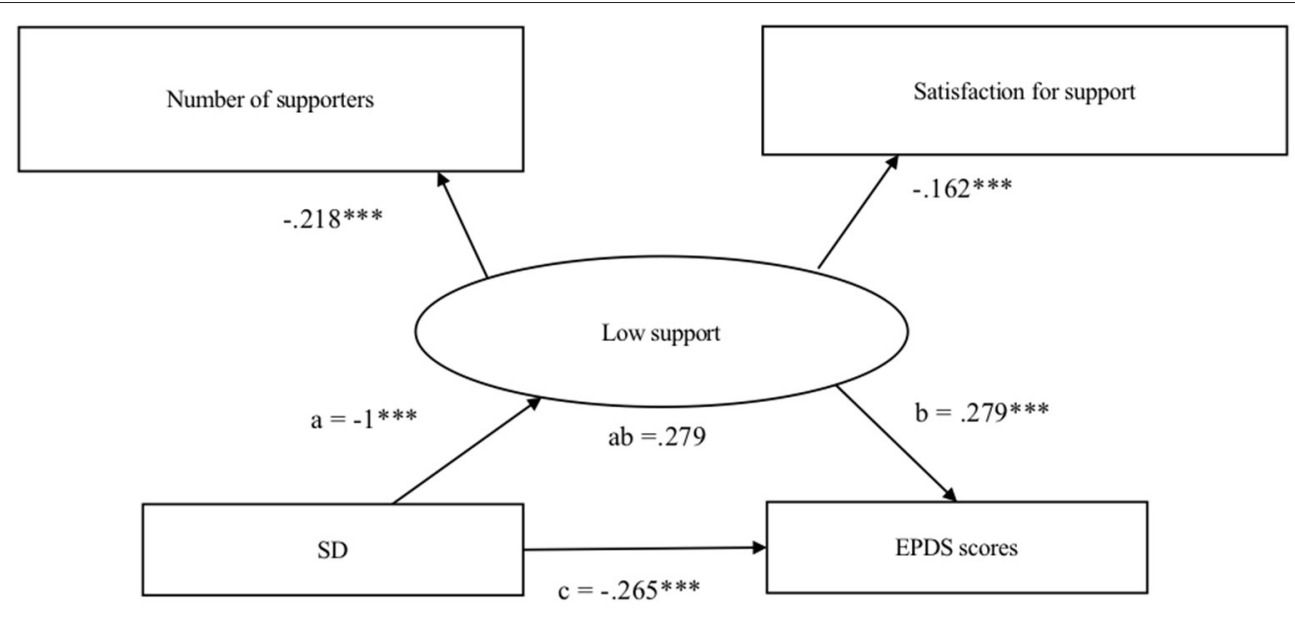

Total model effect $=-.544 * * *$

FIGURE 3 | Structural equation modeling of self-directedness, social support, and postpartum depressive symptoms. Observed measures are represented by squares and latent variables are represented by circles. Single-headed arrows (path) define causal relationships between variables. Numbers represents standardized coefficient. a, effect of SD on low support; b, effect of low support on EPDS total scores; c, direct effect of SD on EPDS total scores; ab, indirect effect of SD on EPDS total scores. SD, self-directedness; EPDS, Edinburgh Postnatal Depression Scale. ${ }^{\star} P<0.05,{ }^{\star \star} P<0.01,{ }^{\star \star \star} P<0.001$.

coping skills. Therefore, it could be considered that subjects with high HA had difficulty in coping with their stressors when using a problem-focused approach, which may have led to not seeking social support or underestimating the actual social support available to them. On the other hand, HA has been reported to be associated with anxiety symptoms (47) and we have previously shown that EPDS has three-factor consisting of anxiety, depression, and anhedonia (48). Future study which examines social support specifically affect these specific factors of EPDS is needed. In contrast, high SD subjects had difficulty in utilizing emotion-focused coping skills (46), and thus their depressive symptoms might be independent of the availability of social support. Intensive psychological care, such as cognitive behavioral therapy, might be effective for those subjects, rather than an increase in social support $(49,50)$.

Some limitations should be considered in this study. First, there is a possible selection bias due to the limited target facilities. In addition, we included only pregnant women who participated in the perinatal class. Second, the variables used in this study were mostly evaluated based on self-report questionnaires, not on a diagnosis by a psychiatrist nor a structured clinical interview. Third, we started evaluating HA, J-SSQ, and EPDS in 2004, while evaluating SD started in 2011. Therefore, the number of samples available for analysis differs between HA and SD, which may have affected the results. Finally, there is a possibility that $\mathrm{HA}$ and $\mathrm{SD}$ itself has been affected by maternal symptoms.

In conclusion, in this study we have shown that social support may work differently with specific personality traits on depressive symptoms after delivery. It is important to provide appropriate support according to mothers' temperament and characteristics to prevent the development of postpartum depression.

\section{DATA AVAILABILITY STATEMENT}

The raw data supporting the conclusions of this article will be made available by the authors, without undue reservation.

\section{ETHICS STATEMENT}

The studies involving human participants were reviewed and approved by the Ethics Committee of the Nagoya University Graduate School of Medicine. The patients/participants provided their written informed consent to participate in this study.

\section{AUTHOR CONTRIBUTIONS}

YN, NT, AY, and NO conceptualized, designed the study, and interpreted the data. YN, NT, AY, TO, MM, and NO acquired and analyzed the data. YN, NT, and NO drafted the manuscript, tables, and figures. All authors approved the final version to be published.

\section{FUNDING}

This work was supported by research grants from the Ministry of Education, Culture, Sports, Science and Technology of Japan; the Ministry of Health, Labor and Welfare of Japan; the Academic Frontier Project for Private Universities, Comparative Cognitive Science Institutes, Meijo University; the Core Research for Evolutional Science and Technology; Intramural Research Grant (21B-2) for Neurological and Psychiatric Disorders from the National Center of Neurology and Psychiatry and the New Technology Development Foundation (the Specific Research Fund 2012 for East Japan Great Earthquake Revival to TO); Research and Development Grants 
for Comprehensive Research for Persons with Disabilities from the Japan Agency for Medical Research and Development (AMED, JP20dk0307077, JP21wm0425007, JP21dm0207075, JP21dk0307103 to NO); JSPS KAKENHI (JP20K07943 to YN and $21 \mathrm{~K} 07479$ to NT).

\section{REFERENCES}

1. O'Hara MW, Swain AM. Rates and risk of postpartum depression-a metaanalysis. Int Rev Psychiatry. (1996) 8:37. doi: 10.3109/09540269609037816

2. Dietz PM, Williams SB, Callaghan WM, Bachman DJ, Whitlock EP, Hornbrook MC. Clinically identified maternal depression before, during, and after pregnancies ending in live births. Am J Psychiatry. (2007) 164:151520. doi: 10.1176/appi.ajp.2007.06111893

3. Woody CA, Ferrari AJ, Siskind DJ, Whiteford HA, Harris MG. A systematic review and meta-regression of the prevalence and incidence of perinatal depression. J Affect Disord. (2017) 219:86-92. doi: 10.1016/j.jad.2017.05.003

4. Takeda S, Takeda J, Murakami K, Kubo T, Hamada H, Murakami M, et al. Annual report of the Perinatology Committee, Japan Society of Obstetrics and Gynecology, 2015: proposal of urgent measures to reduce maternal deaths. $J$ Obstet Gynaecol Res. (2017) 43:5-7. doi: 10.1111/jog.13184

5. Gelaye B, Kajeepeta S, Williams MA. Suicidal ideation in pregnancy: an epidemiologic review. Arch Womens Ment Health. (2016) 19:74151. doi: 10.1007/s00737-016-0646-0

6. Jahan N, Went TR, Sultan W, Sapkota A, Khurshid H, Qureshi IA, et al. Untreated depression during pregnancy and its effect on pregnancy outcomes: a systematic review. Cureus. (2021) 13:e17251. doi: 10.7759/cureus.17251

7. Farias-Antunez S, Xavier MO, Santos IS. Effect of maternal postpartum depression on offspring's growth. J Affect Disord. (2018) 228:143-52. doi: 10.1016/j.jad.2017.12.013

8. Oyetunji A, Chandra P. Postpartum stress and infant outcome: a review of current literature. Psychiatry Res. (2020) 284:112769. doi: 10.1016/j.psychres.2020.112769

9. Morgan JE, Channon S, Penny H, Waters CS. Longitudinal studies examining the impact of prenatal and subsequent episodes of maternal depression on offspring antisocial behaviour. Eur Child Adolesc Psychiatry. (2021) 30:540. doi: 10.1007/s00787-019-01447-w

10. Miguez MC, Vazquez MB. Risk factors for antenatal depression: a review. World J Psychiatry. (2021) 11:325-36. doi: 10.5498/wjp.v11.i7.325

11. Zhao XH, Zhang ZH. Risk factors for postpartum depression: an evidencebased systematic review of systematic reviews and meta-analyses. Asian J Psychiatr. (2020) 53:102353. doi: 10.1016/j.ajp.2020.102353

12. Khazanov GK, Ruscio AM. Is low positive emotionality a specific risk factor for depression? A meta-analysis of longitudinal studies. Psychol Bull. (2016) 142:991-1015. doi: 10.1037/bul0000059

13. Zaninotto L, Solmi M, Toffanin T, Veronese N, Cloninger CR, Correll CU. A meta-analysis of temperament and character dimensions in patients with mood disorders: comparison to healthy controls and unaffected siblings. J Affect Disord. (2016) 194:84-97. doi: 10.1016/j.jad.2015. 12.077

14. Solmi M, Zaninotto L, Toffanin T, Veronese N, Lin K, Stubbs B, et al. A comparative meta-analysis of TEMPS scores across mood disorder patients, their first-degree relatives, healthy controls, and other psychiatric disorders. $J$ Affect Disord. (2016) 196:32-46. doi: 10.1016/j.jad.2016.02.013

15. Wakamatsu M, Nakamura M, Douchi T, Kasugai M, Kodama S, Sano A, et al. Predicting postpartum depression by evaluating temperament during pregnancy. J Affect Disord. (2021) 292:720-4. doi: 10.1016/j.jad.2021.05.106

16. Cloninger CR. A systematic method for clinical description and classification of personality variants. A proposal. Arch Gen Psychiatry. (1987) 44:57388. doi: 10.1001/archpsyc.1987.01800180093014

17. Farmer A, Mahmood A, Redman K, Harris T, Sadler S, McGuffin P. A sib-pair study of the Temperament and Character Inventory scales in major depression. Arch Gen Psychiatry. (2003) 60:490-6. doi: 10.1001/archpsyc.60.5.490

\section{ACKNOWLEDGMENTS}

We would like to acknowledge all participants in the study and the staff of Nagoya Teishin Hospital, Kaseki Hospital, Royal Bell Clinic, and Nagoya University Hospital.

18. Hansenne M, Reggers J, Pinto E, Kjiri K, Ajamier A, Ansseau M. Temperament and character inventory (TCI) and depression. J Psychiatr Res. (1999) 33:316. doi: 10.1016/S0022-3956(98)00036-3

19. Kampman O, Poutanen O. Can onset and recovery in depression be predicted by temperament? A systematic review and meta-analysis. J Affect Disord. (2011) 135:20-7. doi: 10.1016/j.jad.2010.12.021

20. Cloninger CR, Svrakic DM, Przybeck TR. A psychobiological model of temperament and character. Arch Gen Psychiatry. (1993) 50:97590. doi: 10.1001/archpsyc.1993.01820240059008

21. Lim CR, Barlas J, Ho RCM. The effects of temperament on depression according to the schema model: a scoping review. Int J Environ Res Public Health. (2018) 15:1231. doi: 10.3390/ijerph15061231

22. Nemes B, Cozman D. The relevance of personality assessment in estimating the risk of onset and the outcome of major depressive disorder. Clujul Med. (2016) 89:212-5. doi: 10.15386/cjmed-563

23. Hirano S, Sato T, Narita T, Kusunoki K, Ozaki N, Kimura S, et al. Evaluating the state dependency of the Temperament and Character Inventory dimensions in patients with major depression: a methodological contribution. J Affect Disord. (2002) 69:31-8. doi: 10.1016/S0165-0327(00)00329-3

24. Furumura K, Koide T, Okada T, Murase S, Aleksic B, Hayakawa N, et al. Prospective study on the association between harm avoidance and postpartum depressive state in a maternal cohort of Japanese women. PLoS ONE. (2012) 7:e34725. doi: 10.1371/journal.pone.0034725

25. Klein DN, Kotov R, Bufferd SJ. Personality and depression: explanatory models and review of the evidence. Annu Rev Clin Psychol. (2011) 7:26995. doi: 10.1146/annurev-clinpsy-032210-104540

26. Kubota C, Inada T, Shiino T, Ando M, Aleksic B, Yamauchi A, et al. Relation between perinatal depressive symptoms, harm avoidance, and a history of major depressive disorder: a cohort study of pregnant women in Japan. Front Psychiatry. (2019) 10:515. doi: 10.3389/fpsyt.2019.00515

27. Minatani M, Kita S, Ohashi Y, Kitamura T, Haruna M, Sakanashi $\mathrm{K}$, et al. Temperament, character, and depressive symptoms during pregnancy: a study of a Japanese population. Depress Res Treat. (2013) 2013:140169. doi: 10.1155/2013/140169

28. Andriola E, Di Trani M, Grimaldi A, Donfrancesco R. The relationship between personality and depression in expectant parents. Depress Res Treat. (2011) 2011:356428. doi: 10.1155/2011/356428

29. Josefsson A, Larsson C, Sydsjo G, Nylander PO. Temperament and character in women with postpartum depression. Arch Womens Ment Health. (2007) 10:3-7. doi: 10.1007/s00737-006-0159-3

30. Gelabert E, Subira S, Plaza A, Torres A, Navarro P, Imaz ML, et al. The Vulnerable Personality Style Questionnaire: psychometric properties in Spanish postpartum women. Arch Womens Ment Health. (2011) 14:11524. doi: 10.1007/s00737-010-0186-y

31. Morikawa M, Okada T, Ando M, Aleksic B, Kunimoto S, Nakamura $\mathrm{Y}$, et al. Relationship between social support during pregnancy and postpartum depressive state: a prospective cohort study. Sci Rep. (2015) 5:10520. doi: 10.1038/srep 10520

32. Kendler KS, Gardner CO, Prescott CA. Toward a comprehensive developmental model for major depression in women. Am J Psychiatry. (2002) 159:1133-45. doi: 10.1176/appi.ajp.159.7.1133

33. Kijima N, Saito R, Takeuchi M, Yoshino A, Ohno Y, Kato G, et al. Cloninger's seven-factor model of temperament and character and Jananese version of Temperament and Character Inventory (TCI). Arch Psychiatr Diagn Clin Eval. (1996) 7:379-99.

34. Kijima N, Tanaka E, Suzuki N, Higuchi H, Kitamura T. Reliability and validity of the Japanese version of the Temperament and Character Inventory. Psychol Rep. (2000) 86:1050-8. doi: 10.2466/pr0.2000.86.3.1050 
35. Takeuchi M, Miyaoka H, Tomoda A, Suzuki M, Lu X, Kitamura T. Validity and reliability of the Japanese version of the Temperament and Character Inventory: a study of university and college students. Compr Psychiatry. (2011) 52:109-17. doi: 10.1016/j.comppsych.2010.04.002

36. Furukawa TA, Harai H, Hirai T, Kitamura T, Takahashi K. Social Support Questionnaire among psychiatric patients with various diagnoses and normal controls. Soc Psychiatry Psychiatr Epidemiol. (1999) 34:21622. doi: $10.1007 / \mathrm{s} 001270050136$

37. Cox JL, Holden JM, Sagovsky R. Detection of postnatal depression. Development of the 10-item Edinburgh Postnatal Depression Scale. $\mathrm{Br} J$ Psychiatry. (1987) 150:782-6. doi: 10.1192/bjp.150.6.782

38. Okano S, Murata M, Masuji S, Tamaki R, Nomura J, Miyaoka H. Reliability and validity of the Edinburgh postpartum depression scale (EPDS) in Japan. Arch Psychiatr Diagnos Clin Eval. (1996) 7:525-33.

39. Okano T, Murata M, Masuji F, Tamaki R, Nomura J, Miyaoka H, et al. Validation and reliability of Japanese version of the EPDS. Arch Psychiatr Diag Clin Eval. (1996) 7:523-33.

40. Yamashita H, Yoshida K, Nakano H, Tashiro N. Postnatal depression in Japanese women. Detecting the early onset of postnatal depression by closely monitoring the postpartum mood. J Affect Disord. (2000) 58:14554. doi: 10.1016/S0165-0327(99)00108-1

41. Mehmetoglu M. MEDSEM: Stata Module to Perform Mediation Analysis Using Structural Equation Modelling. Boston College Department of Economics (2017).

42. Jose PE. Doing Statistical Mediation and Moderation. London: Guilford (2013).

43. Ohara M, Nakatochi M, Okada T, Aleksic B, Nakamura Y, Shiino $\mathrm{T}$, et al. Impact of perceived rearing and social support on bonding failure and depression among mothers: a longitudinal study of pregnant women. J Psychiatr Res. (2018) 105:71-7. doi: 10.1016/j.jpsychires.2018. 09.001

44. Seymour-Smith M, Cruwys T, Haslam SA. More to lose? Longitudinal evidence that women whose social support declines following childbirth are at increased risk of depression. Aust N Z J Public Health. (2021) 45:33843. doi: 10.1111/1753-6405.13099

45. Mercan Y, Tari Selcuk K. Association between postpartum depression level, social support level and breastfeeding attitude and breastfeeding self-efficacy in early postpartum women. PLoS ONE. (2021) 16:e0249538. doi: 10.1371/journal.pone.0249538
46. Hwang HC, Kim SM, Han DH, Min KJ. Associations of temperament and character with coping strategies among office workers. Psychiatry Investig. (2020) 17:37-46. doi: 10.30773/pi.2019.0084

47. Kampman O, Viikki M, Jarventausta K, Leinonen E. Meta-analysis of anxiety disorders and temperament. Neuropsychobiology. (2014) 69:17586. doi: $10.1159 / 000360738$

48. Kubota C, Okada T, Aleksic B, Nakamura Y, Kunimoto S, Morikawa $\mathrm{M}$, et al. Factor structure of the Japanese version of the Edinburgh Postnatal Depression Scale in the postpartum period. PLoS ONE. (2014) 9:e103941. doi: 10.1371/journal.pone.0103941

49. Anderson CB, Joyce PR, Carter FA, McIntosh VV, Bulik CM. The effect of cognitive-behavioral therapy for bulimia nervosa on temperament and character as measured by the temperament and character inventory. Compr Psychiatry. (2002) 43:182-8. doi: 10.1053/comp.2002.32359

50. Van Lieshout RJ, Layton H, Savoy CD, Brown JSL, Ferro MA, Streiner DL, et al. Effect of online 1-day cognitive behavioral therapybased workshops plus usual care vs usual care alone for postpartum depression: a randomized clinical trial. JAMA Psychiatry. (2021) 78:1200-7. doi: 10.1001/jamapsychiatry.2021.2488

Conflict of Interest: The authors declare that the research was conducted in the absence of any commercial or financial relationships that could be construed as a potential conflict of interest.

Publisher's Note: All claims expressed in this article are solely those of the authors and do not necessarily represent those of their affiliated organizations, or those of the publisher, the editors and the reviewers. Any product that may be evaluated in this article, or claim that may be made by its manufacturer, is not guaranteed or endorsed by the publisher.

Copyright (c) 2022 Nakamura, Takahashi, Yamauchi, Morikawa, Okada and Ozaki. This is an open-access article distributed under the terms of the Creative Commons Attribution License (CC BY). The use, distribution or reproduction in other forums is permitted, provided the original author(s) and the copyright owner(s) are credited and that the original publication in this journal is cited, in accordance with accepted academic practice. No use, distribution or reproduction is permitted which does not comply with these terms. 